\title{
Residuos de plaguicidas y el trastorno del espectro autista
}

\author{
Pesticide residues and autism spectrum disorder
}

\author{
Pedro Andreo-Martínez ${ }^{*}$, Inmaculada Navarro-González ${ }^{2}$, Nuria García-Martínez ${ }^{3}$
}

\begin{abstract}
Citación/Citation: Andreo-Martínez P, NavarroGonzález I, García-Martínez N. Rev Dis Cli Neuro. 2021; (8)1: 39-51. Available from: https://doi.org/10.14198/DCN.19750
\end{abstract}

Recibido/Received: 17/04/2021

Aceptado/Accepted: 20/04/2021

Financiación/Funding: Este estudio no ha recibido financiación.

Conflicto de intereses/Conflicts of Interest: Los autores declaran no tener conflicto de intereses.

(C) 2021 Pedro Andreo-Martínez, Inmaculada Navarro-González y Nuria García-Martínez

Licencia/Licence: This work is licensed under a Creative Commons Attribution 4.0 International License (CC BY 4.0).

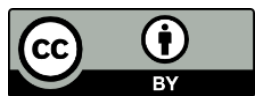

Editorial: Universidad de Alicante (España) Autores/Authors:

${ }^{1}$ Departamento de Química Agrícola, Geología y Edafología. Universidad de Murcia. España, https://orcid.org/0000-0001-9028-231X

2 Área Científica y Técnica de Investigación (ACTI), Sección de Biología Molecular. Universidad de Murcia. España, https://orcid.org/0000-00025894-6400

${ }^{3}$ Departamento de Química Agrícola, Geología y Edafología. Universidad de Murcia. España, https://orcid.org/0000-0001-6535-5492

* Contact for correspondence: pam11@um.es
Resumen: El trastorno del espectro autista (TEA) engloba a un conjunto de trastornos del neurodesarrollo que se caracterizan por una interacción social deficiente, comunicación restringida y comportamientos repetitivos y estereotipados. La etiología del TEA es desconocida a día de hoy, por lo que diversos equipos de investigación multidisciplinares están realizando grandes esfuerzos para intentar dilucidar los factores y mecanismos que intervienen en su aparición. En este sentido, existe un creciente interés en el estudio de factores ambientales como la exposición a residuos de plaguicidas ya que se dispone de suficiente evidencia científica sobre los efectos neurotóxicos que éstos pueden provocar. Por lo tanto, pueden ser formuladas hipótesis de asociaciones específicas entre el TEA y los residuos de plaguicidas. Este trabajo realiza una revisión sistemática sobre la influencia de la exposición a residuos de plaguicidas y la aparición del TEA. La metodología para llevar a cabo este trabajo de revisión sistemática siguió las directrices marcadas por el método PRISMA, encontrándose un total de siete artículos elegibles para su discusión. Los residuos de plaguicidas estudiados por los artículos seleccionados fueron los organofosforados y sus metabolitos; los organoclorados, incluidos el endosulfán y los bifenilos policlorados junto a sus metabolitos; los carbamatos; los piretroides, incluida la cipermetrina; y el glufosinato de amonio. El estudio de la acción de estos plaguicidas se centró en el periodo prenatal, investigándose su exposición en madres gestantes que vivían en zonas próximas a campos de cultivo donde se aplican pesticidas, en modelos animales de ratón y en cultivos de células cerebrales. Se encontró que existe relación entre la exposición a plaguicidas en el periodo prenatal y el riesgo de aparición de TEA en la descendencia junto a una disbiosis en la microbiota intestinal en ratones. Por lo tanto, es importante evaluar el factor de riesgo de exposición a residuos de plaguicidas en la aparición del TEA con más datos, para lo cual se requieren más estudios tanto in vitro como in vivo con el fin de dilucidar los mecanismos bioquímicos precisos involucrados.

Palabras clave: Exposición prenatal; Neurotoxicidad; Residuos de plaguicidas; Revisión Sistemática; TEA. 


\begin{abstract}
Autism Spectrum Disorder (ASD) encompasses a group of neurodevelopmental disorders characterized by poor social interaction, restricted communication, and repetitive and stereotyped behaviors. The etiology of ASD is unknown today, so various multidisciplinary research teams are making great efforts to try to elucidate the factors and mechanisms involved in its appearance. In this sense, there is a growing interest in the study of environmental factors such as exposure to pesticide residues since there is sufficient scientific evidence on the neurotoxic effects that these can cause. Therefore, specific associations between TEA and pesticide residues can be hypothesized. This work carries out a systematic review on the influence of exposure to pesticide residues and the appearance of ASD. The methodology to carry out this systematic review followed the guidelines set by the PRISMA method, finding a total of seven articles eligible for discussion. The pesticide residues studied by the selected articles were organophosphates and their metabolites; organochlorines, including endosulfan and polychlorinated biphenyls together with their metabolites; carbamates; pyrethroids, including cypermethrin; and glufosinate ammonium. The study of the action of these pesticides focused on the prenatal period, investigating their exposure in pregnant mothers who lived in areas close to fields where pesticides are applied, in mouse animal models and in brain cell cultures. It was found that there is a relationship between exposure to pesticides in the prenatal period and the risk of the appearance of ASD in the offspring together with a dysbiosis of the gut microbiota in mice. Therefore, it is important to evaluate the risk factor of exposure to pesticide residues in the appearance of ASD with more data, for which more studies are required both in vitro and in vivo in order to elucidated the accurate biochemical mechanisms involved.
\end{abstract}

Keywords: Autism; Neurotoxicity; Pesticide residues; Prenatal exposure; Systematic Review.

\title{
1. Introducción
}

El Trastorno del Espectro Autista (TEA) es un trastorno del neurodesarrollo que se manifiesta durante los primeros tres años de vida y persiste durante toda la vida del individuo. El Manual Diagnóstico y Estadístico de los Trastornos Mentales DSM-5 define el TEA como un grupo heterogéneo de trastornos del neurodesarrollo que se caracterizan por alteraciones en la comunicación, trastornos de interacción social y comportamientos repetitivos [1]. El TEA también incluye el autismo de Kanner [2], el síndrome de Asperger [3] y el trastorno generalizado del desarrollo no especificado de otra manera. El TEA es más prevalente en hombres que en mujeres (4:1) y su incidencia experimentó un drástico aumento desde principios de la década de los 90, que puede ser atribuible a cambios en el diagnóstico de la enfermedad [4]. Se estima que en España 8 de cada 10.000 niños padecen TEA [5], y se piensa que las personas con este trastorno tienen una mala calidad de vida y que son una pesada carga para la sociedad $[6,7]$.

La etiología del TEA es desconocida a día de hoy, por lo que diversos equipos de investigación multidisciplinares están realizando grandes esfuerzos para intentar dilucidar los factores y mecanismos que intervienen en su aparición. En este sentido, todo apunta, aparentemente, a que las complejas interacciones entre factores genéticos, epigenéticos y ambientales pueden contribuir al desarrollo y 
expresión del TEA [8,9]. Muchas hipótesis implican un déficit funcional causado por alteraciones en estructuras cerebrales específicas que ocurren en el útero durante ventanas temporales definidas de vulnerabilidad [10]. Por ejemplo, se ha descubierto que una mutación del gen GRIN2B en el cerebro puede estar involucrada en la aparición de TEA [11]. Otra hipótesis apunta a una disbiosis de la microbiota intestinal (MI) de las personas que padecen TEA ya que la MI excreta metabolitos capaces de atravesar la barrera hematoencefálica y ejercer su acción en el cerebro [12]. Cabe destacar que estudios recientes indican que niños con TEA presentan una abundancia relativa estadísticamente significativa más baja de bacterias pertenecientes al género Bifidobacterium ( $S M D+=-0,513 ; 95 \% C I=-0,953,-0,073$ ) comparados con sus grupos control neurotípicos [13]. Con respecto a otros factores ambientales como los residuos de plaguicidas, se ha experimentado un creciente interés en los últimos años por el estudio de los efectos neurotóxicos del desarrollo que éstos pueden provocar ya que existen evidencias de asociaciones específicas entre el TEA y los pesticidas agrícolas [14, 15].

Los pesticidas son una categoría de productos químicos tóxicos que están formulados para matar o repeler plagas o para interrumpir su reproducción [16, 17], donde se incluyen insecticidas, acaricidas, alguicidas, herbicidas y fungicidas, y son algunas de las sustancias más tóxicas, ambientalmente estables y móviles del medio ambiente [18]. Los insecticidas y acaricidas matan insectos y ácaros al interrumpir su actividad neuronal, el proceso de muda u otros mecanismos específicos del metabolismo de estos artrópodos. Los alguicidas y herbicidas matan plantas y algas al alterar las capacidades fotosintéticas o la síntesis de compuestos orgánicos esenciales, y los fungicidas matan a los hongos inhibiendo, por ejemplo, la formación de membranas celulares en dichos organismos [19].

La exposición del ser humano a los residuos de plaguicidas se debe, principalmente, a la contaminación de la cadena alimentaria (agua, pescado, carne, etc.), la inhalación de polvo doméstico contaminado o la exposición ocupacional. Estos contaminantes ambientales interactúan con las hormonas y pueden producir consecuencias adversas debido a su acción de disrupción endocrina [20]. Su toxicidad tanto en órganos como en tejidos depende de la dosis, el modo de contacto, los cambios biológicos y el suministro de metabolitos asociados [21].

Los residuos de plaguicidas junto a muchos otros tóxicos ambientales se transmiten a través de la placenta y la barrera hematoencefálica que permanece relativamente permeable a muchos de estos compuestos hasta bien entrado el primer año de vida [15]. Estudios epidemiológicos recientes han demostrado que la exposición a pesticidas en etapas tempranas de la vida está asociada con defectos del neurodesarrollo infantil y trastornos mentales, incluidos el TEA, la disminución del coeficiente intelectual o discapacidades cognitivas del desarrollo. En este sentido, el contacto prenatal a pesticidas podría inducir trastornos del comportamiento, así como aberraciones neuroanatómicas y funcionales [6].

El sistema nervioso central (SNC) de los mamíferos pasa por varias etapas de desarrollo antes de alcanzar la maduración completa. Una fase crítica en el desarrollo del cerebro es la aceleración del crecimiento cerebral (ACC) que se caracteriza por un rápido aumento del tamaño y cambios bioquímicos del cerebro. En los seres humanos, esta fase comienza alrededor del tercer trimestre de embarazo, alcanza su punto máximo alrededor del nacimiento y continúa hasta los dos primeros años de vida. Los procesos bioquímicos que tienen lugar durante la ACC son el establecimiento de conexiones neuronales, sinaptogénesis, poda neuronal y mielinización, entre otros. Estos procesos involucran la expresión y regulación de neuroproteínas como las quinasas II dependientes de calcio / calmodulina (CaMKII), 
proteína 43 asociada al crecimiento (GAP-43), receptor de glutamato 1 (GluR1), proteína de densidad postsináptica 95 (PSD95), sinaptofisina y tau. También se ha demostrado que estas proteínas expresan patrones ontogenéticos específicos durante el período posnatal en el desarrollo del SNC en modelos animales. La ACC es sensible a las agresiones tóxicas de xenobióticos como los éteres difenílicos polibromados (PBDE), bifenilos policlorados (PCB), compuestos perfluorados (PFC), productos farmacéuticos, nicotina y bisfenol A, debido a que estos xenobióticos afectan el nivel de expresión de neuroproteínas cuando se exponen durante esta fase crítica. Además, estudios previos sobre piretroides y organoclorados han demostrado causar neurotoxicidad en el neurodesarrollo de ratones, que se manifiesta como un comportamiento espontáneo alterado y cambios en la densidad del receptor muscarínico de acetilcolina en el cerebro, cuando se expone neonatalmente [14]. Por tanto, el efecto de la exposición a residuos de plaguicidas en el desarrollo neurológico de la descendencia es un tema importante de salud pública.

\section{Objetivo}

El objetivo de este trabajo es describir la relación existente entre los residuos de plaguicidas y la etiología del TEA haciendo uso de las directrices PRISMA 2020.

\section{Método}

Para realizar el estudio de revisión sistemática sobre residuos de plaguicidas y TEA se siguieron las directrices marcadas por el protocolo PRISMA [22], donde se describe la metodología de búsqueda bibliográfica para obtener las publicaciones científicas más relevantes con el fin de responder a la pregunta clave objeto de investigación. Las cuatro bases de datos elegidas fueron Web of Science, Scopus, PubMed y Science Database, utilizando las palabras clave "pesticide residue*" AND “autism OR ASD". Las opciones de búsqueda en las bases de datos fueron: "theme" en la Web of Science, "Article title, Abstract, Keyword" en Scopus, "todos los campos" en PubMed y "todos los campos menos texto completo (NOFT)" en Science Database. El periodo de tiempo seleccionado fue todos los años hasta el 6 de marzo de 2021. Los criterios de inclusión fueron: artículos sobre residuos de plaguicidas y TEA y artículos publicados hasta el 6 de marzo de 2021. Los criterios de exclusión fueron: revisiones, congresos, libros y capítulos de libro, cartas al editor o material editorial, y artículos que no estudien las implicaciones de los residuos de plaguicidas en el TEA. El programa de gestión bibliográfico EndNote X9 fue utilizado para detectar posibles duplicados en las publicaciones obtenidas por las bases de datos y el equipo revisor estuvo formado por las autoras Inmaculada Navarro-González y Nuria García-Martínez. Por último, se realizó una evaluación de calidad de los artículos seleccionados, donde se valoraron los riesgos de sesgo de cada uno de ellos $[12,23]$.

\section{Resultados}

Utilizando los criterios de búsqueda descritos en la sección "Método", trece, tres, dos y dos publicaciones fueron encontradas por las bases de datos Web of Science, Scopus, Science Database y PubMed, 
respectivamente. Cuatro publicaciones duplicadas fueron eliminadas utilizando el software EndNote X9. De las 16 referencias restantes, siete revisiones, una conferencia y un libro editorial fueron eliminadas en el proceso de cribado, revisándose siete artículos a texto completo para evaluar su elegibilidad. Entre esos siete artículos se encontró un "erratum" y un artículo que solo hablaba de los plaguicidas en la agricultura. Además, dos artículos elegibles fueron encontrados revisando la bibliografía de los artículos preseleccionados [15, 24]. Finalmente, se encontró que siete artículos cumplieron con los criterios de inclusión establecidos para la presente revisión sistemática (Figura 1).

Figura 1. Diagrama de flujo de los artículos seleccionados.

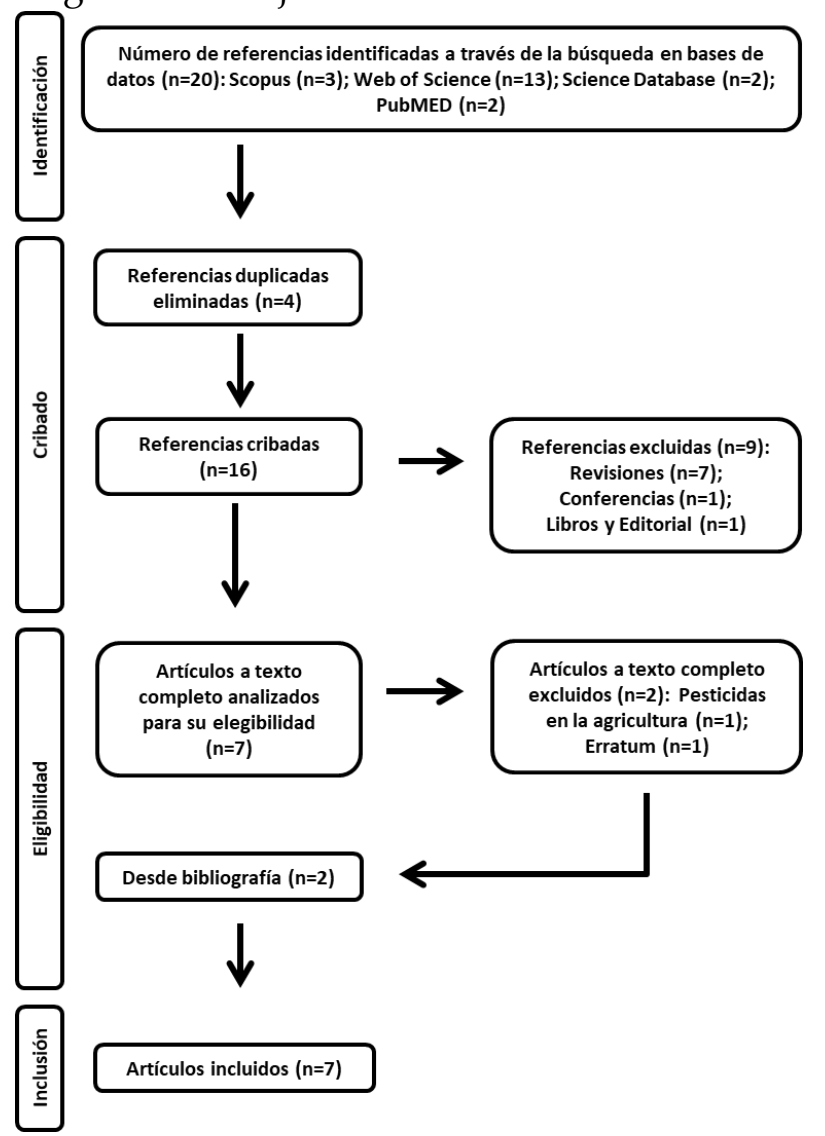

La Tabla 1 muestra las principales características de los siete artículos seleccionados. Como puede ser observado, cuatro artículos (57,1 \%) fueron estudios retrospectivos causa-efecto [15, 25, 26], dos artículos $(28,5 \%)$ realizaron sus investigaciones utilizando un modelo animal de ratón [6,14], y un artículo $(14,3 \%)$ utilizó un modelo de cultivos celulares [27]. Los cuatro estudios retrospectivos causa-efecto estudiaron el riesgo prenatal de residir en zonas cercanas a cultivos agrícolas donde se aplican plaguicidas. Los residuos de plaguicidas estudiados fueron los organofosforados y sus metabolitos; los organoclorados, incluidos el endosulfán y los bifenilos policlorados junto a sus metabolitos; los carbamatos; los piretroides, incluida la cipermetrina; y el glufosinato de amonio. 
Tabla 1. Objetivo, tipo de estudio, plaguicidas y principales resultados encontrados en los 7 artículos seleccionados en la presente revisión sistemática.

\begin{tabular}{|c|c|c|c|c|}
\hline Referencia & Objetivo & $\begin{array}{l}\text { Tipo } \\
\text { estudio }\end{array}$ & Plaguicida/s & Principales resultados \\
\hline [6] & $\begin{array}{l}\text { Efecto de la } \\
\text { exposición prenatal } \\
\text { del glufosinato de } \\
\text { amonio sobre el } \\
\text { comportamiento }\end{array}$ & $\begin{array}{l}\text { Modelo } \\
\text { animal }\end{array}$ & $\begin{array}{l}\text { Glufosinato } \\
\text { amonio }\end{array}$ & $\begin{array}{l}\text { Actividad locomotora } \\
\text { reducida, formación de } \\
\text { memoria alterada y } \\
\text { comportamientos } \\
\text { similares al TEA }\end{array}$ \\
\hline [24] & $\begin{array}{l}\text { Efecto de la } \\
\text { exposición prenatal } \\
\text { de pesticidas } \\
\text { organofosforados y } \\
\text { riesgo de TEA }\end{array}$ & $\begin{array}{l}\text { Estudio } \\
\text { retrospectivo } \\
\text { causa-efecto o } \\
\text { retrospectivo } \\
\text { caso-control }\end{array}$ & Organofosforados & $\begin{array}{l}\text { Asociaciones de factores } \\
\text { de riesgo ambientales } \\
\text { modificables con rasgos } \\
\text { relacionados con el TEA }\end{array}$ \\
\hline [25] & $\begin{array}{l}\text { Efecto combinado } \\
\text { de la exposición } \\
\text { prenatal a pesticidas } \\
\text { y el consumo de } \\
\text { ácido fólico con el } \\
\text { riesgo de TEA }\end{array}$ & $\begin{array}{l}\text { Estudio } \\
\text { retrospectivo } \\
\text { causa-efecto o } \\
\text { retrospectivo } \\
\text { caso-control }\end{array}$ & $\begin{array}{l}\text { Organofosforados } \\
\text { Piretroides } \\
\text { Carbamatos }\end{array}$ & $\begin{array}{l}\text { El consumo de ácido } \\
\text { fólico durante el primer } \\
\text { mes de embarazo podría } \\
\text { reducir el riesgo de TEA } \\
\text { asociado a la exposición } \\
\text { materna a pesticidas } \\
\text { antes y durante el } \\
\text { embarazo }\end{array}$ \\
\hline [14] & $\begin{array}{lr}\text { Posibles } & \text { efectos } \\
\text { neurotóxicos } & \text { de } \\
\text { endosulfán } & y \\
\text { cipermetrina } & \text { en } \\
\text { la } \\
\text { exposición neonatal }\end{array}$ & $\begin{array}{l}\text { Modelo } \\
\text { animal }\end{array}$ & $\begin{array}{l}\text { Endosulfán } \\
\text { Cipermetrina }\end{array}$ & $\begin{array}{lr}\text { Niveles alterados de } \\
\text { neuroproteínas } \\
\text { alteraciones } \\
\text { comportamiento } \\
\text { compatibles con el TEA }\end{array}$ \\
\hline [26] & $\begin{array}{l}\text { Trastornos del } \\
\text { neurodesarrollo y } \\
\text { proximidad } \\
\text { residencial prenatal } \\
\text { a pesticidas }\end{array}$ & $\begin{array}{l}\text { Estudio } \\
\text { retrospectivo } \\
\text { causa-efecto } \\
\text { o } \\
\text { retrospectivo } \\
\text { caso-control }\end{array}$ & $\begin{array}{l}\text { Organofosforados } \\
\text { Organoclorados } \\
\text { Piretroides } \\
\text { Carbamatos }\end{array}$ & $\begin{array}{l}\text { Vinculación evidenciada } \\
\text { de los trastornos del } \\
\text { desarrollo neurológico } \\
\text { con la exposición a } \\
\text { plaguicidas durante la } \\
\text { gestación }\end{array}$ \\
\hline [27] & $\begin{array}{l}\text { Efecto de los PCBs } \\
\text { sobre el desarrollo } \\
\text { de desórdenes } \\
\text { cerebrales }\end{array}$ & $\begin{array}{l}\text { Cultivos } \\
\text { celulares } \\
\text { cerebrales }\end{array}$ & PCBs & $\begin{array}{l}\text { PCBs suprimen el } \\
\text { desarrollo de las } \\
\text { neuronas de Purkinje }\end{array}$ \\
\hline [15] & $\begin{array}{l}\text { Riesgo de TEA de la } \\
\text { descendencia de } \\
\text { mujeres } \\
\text { embarazadas que } \\
\text { residen en zonas } \\
\text { cercanas donde se } \\
\text { aplican plaguicidas }\end{array}$ & $\begin{array}{l}\text { Estudio } \\
\text { retrospectivo } \\
\text { causa-efecto } \\
\text { o } \\
\text { retrospectivo } \\
\text { caso-control }\end{array}$ & Organoclorados & $\begin{array}{l}\text { El riesgo de TEA y la } \\
\text { proximidad a zonas de } \\
\text { aplicación de plaguicidas } \\
\text { aumentó la dosis y se } \\
\text { atenuó al aumentar la } \\
\text { distancia de residencia a } \\
\text { la zona de aplicación }\end{array}$ \\
\hline
\end{tabular}


Cuatro de los 7 artículos seleccionados se publicaron en la revista científica Environmental Health Perspectives, uno en Journal of Hazardous Materials, uno en Chemposphere y otro en Toxicology. Cuatro artículos fueron publicados en Estados Unidos, uno en China, uno en Japón y uno en Suiza. Los artículos se publicaron entre 2007 y 2020.

La Tabla 2 muestra la puntuación asignada para los diferentes tipos de sesgo. La evaluación de la calidad de los siete artículos seleccionados mostró que todos los artículos presentaban un riesgo de sesgo bajo, tomando en consideración que los artículos presentaban un riesgo de sesgo alto, moderado o bajo en términos de una puntuación consolidada sobre 12.

Tabla 2. Evaluación de la calidad de los 7 artículos seleccionados en la presente revisión sistemática.

\begin{tabular}{|c|c|c|c|c|c|c|c|}
\hline Artículo & [6] & [27] & [14] & [25] & [26] & [15] & [24] \\
\hline Objetivo claramente establecido & 2 & 2 & 2 & 2 & 2 & 2 & 2 \\
\hline Tamaño del estudio apropiado & 2 & 2 & 2 & 2 & 2 & 2 & 2 \\
\hline Identificación y evaluación de la muestra & 2 & 2 & 2 & 2 & 2 & 2 & 2 \\
\hline Comparabilidad & 2 & 2 & 2 & 2 & 2 & 2 & 2 \\
\hline $\begin{array}{l}\text { Otros sesgos (datos de exposición previa, otros } \\
\text { factores medioambientales ...) }\end{array}$ & 2 & 0 & 2 & 2 & 2 & 2 & 2 \\
\hline Análisis estadístico adecuado & 2 & 2 & 2 & 2 & 2 & 2 & 2 \\
\hline TOTAL & 12 & 10 & 12 & 12 & 12 & 12 & 12 \\
\hline Riesgo de sesgo & 0 & 2 & 0 & 0 & 0 & 0 & 0 \\
\hline Riesgo de sesgo general & B & B & B & B & $\mathrm{B}$ & B & $\mathrm{B}$ \\
\hline
\end{tabular}

Nota: 0 = No informa o evalúa; 1 = no evaluado adecuadamente; 2 = adecuadamente evaluado; $\mathrm{M}=$ Medio (7-9); B= Bajo (10-12); A = Alto (1-6)

Las principales limitaciones de este estudio de revisión sistemática fueron las bases de datos elegidas, los criterios de inclusión / exclusión establecidos y los términos de búsqueda seleccionados. En este sentido, cabe destacar que dos artículos (28,5\%) que cumplían los criterios de inclusión, encontrados en el proceso de revisión de la bibliografía de los artículos seleccionados, no fueron indexados introduciendo las palabras clave elegidas en las diferentes bases de datos seleccionadas. Finalmente, debido al pequeño número de estudios y a la no homogeneización de datos, no pudo realizarse un metaanálisis de los datos de los siete artículos seleccionados [28].

\section{Discusión}

La mayoría de los residuos de plaguicidas son propensos a mantenerse suspendidos en el aire a la "deriva" y los niveles detectables en muestras de aire, a menudo, son significativos en ubicaciones más allá del lugar de aplicación durante prolongados períodos de tiempo [29]. Prueba de ello son los elevados niveles 
de pesticidas encontrados en el polvo doméstico y sus metabolitos en la orina de personas que viven en zonas cercanas a campos de cultivo donde se aplican plaguicidas [30], siendo estos residuos de plaguicidas especialmente neurotóxicos durante el ACC. En este sentido, todos los artículos encontrados en este estudio de revisión sistemática se centran en la exposición prenatal a residuos de plaguicidas y el riesgo de aparición del TEA en madres gestantes que vivían en zonas próximas a campos de cultivo donde se aplican pesticidas, en modelos animales de ratón y en cultivos de células cerebrales. A continuación, se describen los principales plaguicidas que han sido relacionados con el TEA junto a los posibles mecanismos bioquímicos responsables.

\subsection{Glufosinato de amonio}

El glufosinato de amonio (GLA) es un herbicida que se utiliza ampliamente en todo el mundo [31]. Un estudio realizado por Aris and Leblanc [32] encontró que un metabolito del GLA (ácido 3metilfosfinicopropiónico) podría detectarse en casi todas las muestras de suero sanguíneo de un grupo de pares madre-hijo, lo que indica una exposición universal y una potencial exposición transplacentaria. Aunque, la neurotoxicidad del GLA está bien documentada [31, 33], los mecanismos subyacentes a través de los cuales GLA impactan el desarrollo del SNC siguen siendo desconocidos en gran parte. En este sentido, Dong, Guan [6] utilizaron un modelo animal de ratón para estudiar los efectos de la exposición prenatal del GLA. El grupo de tratamiento mostró una actividad locomotora reducida, una formación de memoria alterada y comportamientos similares al TEA. Además, se observó una alteración en la MI y el metabolismo, posibles responsables de los comportamientos anómalos compatibles con el TEA en los ratones.

\subsection{El organoclorado endosulfán y el piretroide cipermetrina}

El endosulfán es un plaguicida restringido, aunque todavía se detecta en productos alimenticios a concentraciones que oscilan entre $<0,1$ y 100 ppb, siendo la principal vía de exposición la ingesta dietética [34]. En el organismo, el endosulfán se encuentra a concentraciones más altas en los riñones y el hígado que en el cerebro. Actúa como un antagonista no competitivo del ácido gamma-aminobutírico (GABA) en los canales de cloro dentro del cerebro [35]. GABA es un neurotransmisor en gran parte exclusivo de las interneuronas, y la actividad mediada por GABA regula la migración celular, la proliferación, la sinaptogénesis y, por extensión, el patrón general de las redes neuronales. Aunque pocos estudios han investigado los efectos neurotóxicos del endosulfán y las investigaciones realizadas no se consideran concluyentes [36], se sabe que la neurotransmisión mediada por GABA desempeña un papel importante en el desarrollo del cerebro gestacional por lo que el metabolismo alterado del GABA podría desempeñar un importante rol en la aparición del TEA [37]. Por otro lado, la cipermetrina es un piretroide de tipo II que causa coreoatetosis (movimientos incontrolados e involuntarios en varias zonas corporales) y salivación después de una exposición aguda. La principal vía de exposición es a través de la ingesta dietética, aunque también se produce exposición cutánea y por inhalación. Las concentraciones tisulares más altas de cipermetrina se encuentran en la grasa corporal, la piel, el hígado, los riñones, las glándulas suprarrenales y los ovarios, mientras que en el cerebro solo se encuentran niveles insignificantes. Aunque varios estudios han investigado los posibles efectos neurotóxicos en el desarrollo de los piretroides, todavía se desconoce la relación bioquímica de este residuo de plaguicida con la conducta [38]. En este sentido, Lee, Eriksson [14] investigaron los posibles efectos neurotóxicos de una sola exposición neonatal de endosulfán y cipermetrina durante el ACC utilizando un modelo animal de ratón. Los resultados 
indicaron que ambos pesticidas pueden inducir niveles alterados de neuroproteínas, importantes para el desarrollo normal del cerebro, y anomalías neuroconductuales que se manifiestan como una conducta adulta espontánea alterada y baja capacidad de habituarse a un nuevo entorno. Los efectos neurotóxicos en el comportamiento estuvieron presentes varios meses después de la prueba inicial, lo que indica efectos irreversibles duraderos o incluso persistentes.

\subsection{Los organoclorados bifenilos policlorados}

Otro tipo de residuo de plaguicida organoclorado son los PCBs y parece ser que los tejidos cerebrales están mejor protegidos contra la acumulación de PCBs que otros tejidos ya que dispone de la barrera hematoencefálica. Sin embargo, los PCBs pueden penetrar fácilmente la barrera hematoencefálica durante las etapas embrionaria y perinatal. Es conocido que los PCBs y sus metabolitos [(hidroxi-PCBs (HO-PCBs)] poseen estructuras químicas similares a la de las hormonas tiroideas (TH), como la tiroxina (T4) y la triyodotironina (T3), y la deficiencia de TH durante el período perinatal provoca cretinismo, acompañado de graves déficits cognitivos y retraso mental en niños. En este sentido, varios experimentos en modelo animal han indicado que los PCBs y compuestos relacionados pueden afectar las funciones de TH y causar daño cerebral [39], ya que algunos PCBs y OH-PCBs pueden interferir potencialmente con la expresión génica mediada por el receptor $\mathrm{TH}$, incluso a concentraciones del orden de $10^{-10} \mathrm{M}$ [40]. Kimura-Kuroda, Nagata [27] estudiaron el efecto de la exposición de PCBs para evaluar la posible aparición de desórdenes cerebrales utilizando cultivos de células cerebrales de ratón. Encontraron que los PCBs suprimían el desarrollo de las neuronas de Purkinje incluso a concentraciones $10^{-11} \mathrm{M}$. Estos hallazgos sugieren que la exposición a PCB y otras sustancias químicas ambientales pueden interrumpir el desarrollo neuronal normal y causar algunos trastornos del neurodesarrollo, donde se incluye el TEA.

\subsection{Otros residuos de plaguicidas encontrados en las proximidades de zonas residenciales}

Los pesticidas organoclorados engloban a un grupo diverso de compuestos químicos derivados de halobenceno utilizados principalmente como insecticidas. Incluyen a dicofol, PCBs y endosulfán, descritos anteriormente. En general, los pesticidas organoclorados se metabolizan a través del sistema del citocromo P450 en humanos. El dicofol es químicamente similar al diclorodifeniltricloroetano (DDT), con la diferencia de que el dicofol posee un resto hidroxi en uno de sus dos átomos de carbono alifáticos. El dicofol no se metaboliza a diclorodifenildicloroetileno (DDE), se elimina más rápidamente y se bioacumula menos que el DDT [41]. Roberts, English [15] evaluaron la hipótesis de que el riesgo de residir en zonas cercanas a cultivos agrícolas donde se aplican pesticidas, durante períodos clave de gestación, podría estar asociado con el desarrollo del TEA en la descendencia. El riesgo de TEA se asoció con la proximidad residencial a las aplicaciones de plaguicidas organoclorados que se producían alrededor del período de embriogénesis del SNC. Esta asociación pareció aumentar con la dosis y disminuyó al aumentar la distancia de residencia del campo de aplicación. Estos hallazgos sugieren la posibilidad de una conexión entre la exposición gestacional a pesticidas organoclorados y la aparición del TEA.

Los OP y carbamatos son un amplio grupo de productos químicos considerados neurotoxinas [42], que se utilizan tanto para fines domésticos como agrícolas, donde se incluyen insecticidas, gases nerviosos, antihelmínticos, y ciertos herbicidas. Los OP inactivan la acetilcolinesterasa (AChE). Una vez inactivada la $\mathrm{AChE}$, la acetilcolina ( $\mathrm{ACh}$ ) se acumula en todo el sistema nervioso autónomo, el sistema nervioso somático y el cerebro, lo que resulta en una sobreestimulación de los receptores muscarínicos y nicotínicos del cerebro. Las neuronas preganglionares y posganglionares del sistema nervioso parasimpático también 
liberan ACh. En este sentido, se sabe que las vías colinérgicas en el cerebro están asociadas con varios tipos de comportamiento y funciones como el hambre, la sed, la termorregulación, la respiración, la agresión y la cognición [43].

Shelton, Geraghty [26] evaluaron si la proximidad de la residencia de madres embarazadas a campos donde se aplican pesticidas está asociada con el TEA o retraso en el desarrollo. Concluyeron que las madres de niños con TEA estuvieron expuestas a pesticidas organofosforados, piretroides y carbamatos durante la gestación, lo que puede suponer un mayor riesgo de trastornos del desarrollo neurológico incluido el TEA.

En esta misma línea, Schmidt, Kogan [25] estudiaron el efecto combinado de la exposición a pesticidas y el consumo de suplementos de ácido fólico en mujeres embarazadas y el riesgo de la aparición del TEA en su descendencia. Estos investigadores concluyeron que, aunque no se pueden descartar explicaciones no causales para la reducción del riesgo de TEA en asociación con la exposición a pesticidas por el ácido fólico, se puede especular que los mecanismos potenciales podrían involucrar las propiedades antioxidantes de los folatos, su papel en la reparación del ADN o su influencia en la metilación del ADN. El papel del ácido fólico como donante de grupos metilo podría ser relevante dado que todas las demás vías propuestas podrían conducir al agotamiento de los grupos metilo necesarios para la metilación del ADN, lo que podría ser crítico cerca de la concepción del neonato, cuando el metiloma se desmetila y luego se restablece. Estos mismos autores también sugirieron que la administración de suplementos de ácido fólico durante el primer mes de embarazo podría reducir, pero no eliminar, el mayor riesgo de TEA asociado con la exposición materna a pesticidas antes y durante el embarazo.

Por último, Sagiv, Harris [24] investigaron la asociación de la exposición de madres embarazadas a plaguicidas OP con el riesgo de aparición de TEA en el neonato. Para ello, realizaron mediciones de los metabolitos dialquilfosfatos en la orina de las madres embarazadas y la relacionaron con la proximidad de su residencia a campos de cultivo agrícolas donde se aplicaban plaguicidas. Encontraron que la presencia de metabolitos urinarios de los pesticidas OP se asociaron con un aumento del riesgo de TEA en la descendencia. Estos hallazgos contribuyen a la evidencia de asociaciones de factores de riesgo ambientales modificables con rasgos relacionados con el TEA.

\section{Conclusiones}

Este estudio de revisión sistemática encontró siete artículos que relacionaban la exposición a residuos de plaguicidas con la aparición del TEA. Con los datos que se barajan hoy en día, la etiología del TEA puede ser considerada como una enfermedad multifactorial donde la exposición a residuos de plaguicidas como factor medioambiental juega un importante papel debido a su carácter neurotóxico y a los recientes descubrimientos de los efectos adversos que provocan sobre el neurodesarrollo. En este sentido, cabe destacar que todos los estudios encontrados en este estudio de revisión sistemática se centran en la exposición prenatal a residuos de plaguicidas y el riesgo de aparición del TEA. Estos estudios encontraron que existe relación entre la exposición a plaguicidas en el periodo prenatal y el riesgo en la descendencia de padecer TEA, y una disbiosis de la MI en ratones. Es importante identificar la etiología de los desórdenes de neurodesarrollo como el TEA para poder implementar mejores medidas de prevención y control. Como ya se ha comentado, los productos químicos ambientales son factores de riesgo importantes 
que están asociados con la aparición de desórdenes del neurodesarrollo además de las anomalías genéticas. Sin embargo, aclarar el papel de los productos químicos ambientales que interfieren con el neurodesarrollo no es fácil debido a la multiplicidad y modificabilidad del medio ambiente. Por lo tanto, es importante evaluar factores de riesgo precisos con más datos, para lo cual se requieren más experimentos en estudios tanto in vitro como in vivo.

\section{Referencias}

1. APA. American Psychiatric Association. Autism spectrum disorder. Diagnostic and Statistical Manual of Mental Disorders, 5 Eds (DSM-5). Washington, DC, USA: American Psychiatric Publishing; 2013.

2. Kanner L. Autistic disturbances of affective contact. Nervous child 1943, 2 (3): 217-250.

3. Asperger H. Die „Autistischen Psychopathen” im Kindesalter. Eur Arch Psychiatry Clin Neurosci 1944, 117 (1): 76-136.

4. Shattuck PT. The Contribution of Diagnostic Substitution to the Growing Administrative Prevalence of Autism in US Special Education. Pediatrics 2006, 117 (4): 1028-1037. doi: https://doi.org/10.1542/peds.2005-1516

5. Andreo-Martínez P, García-Martínez N, Sánchez-Samper EP, Quesada-Medina J, MacFabe D. Metabolites of the gut microbiota involved in the autism spectrum disorder. Rev Dis Cli Neuro 2018, 5 (2): 39-48. doi: https://doi.org/10.14198/DCN.2018.5.2.05

6. Dong T, Guan Q, Hu W, Zhang M, Zhang Y, Chen M, et al. Prenatal exposure to glufosinate ammonium disturbs gut microbiome and induces behavioral abnormalities in mice. J Hazard Mater 2020, 389: 122152. doi: https://doi.org/10.1016/j.jhazmat.2020.122152

7. Wanke KA, Devanna P, Vernes SC. Understanding Neurodevelopmental Disorders: The Promise of Regulatory Variation in the $3^{\prime}$ UTRome. Biol Psychiatry 2018, 83 (7): 548-557. doi: https://doi.org/10.1016/j.biopsych.2017.11.006

8. MacFabe DF. Enteric short-chain fatty acids: microbial messengers of metabolism, mitochondria, and mind: implications in autism spectrum disorders. Microb Ecol Health Dis 2015, 26: 28177. doi: https://doi.org/10.3402/mehd.v26.28177

9. Argou-Cardozo I, Zeidán-Chuliá F. Clostridium Bacteria and Autism Spectrum Conditions: A Systematic Review and Hypothetical Contribution of Environmental Glyphosate Levels. Med Sci 2018, 6 (2): 29. doi: https://doi.org/10.3390/medsci6020029

10. Polleux F, Lauder JM. Toward a developmental neurobiology of autism. Ment Retard Dev Disabil Res Rev 2004, 10 (4): 303-317. doi: https://doi.org/10.1002/mrdd.20044

11. Bell S, Maussion G, Jefri M, Peng H, Theroux J-F, Silveira H, et al. Disruption of GRIN2B Impairs Differentiation in Human Neurons. Stem Cell Reports 2018, 11 (1): 183-196. doi: https://doi.org/10.1016/j.stemcr.2018.05.018

12. Andreo-Martínez P, García-Martínez N, Sánchez-Samper EP, Martínez-González AE. An approach to gut microbiota profile in children with autism spectrum disorder. Environ Microbiol Rep 2019, 12 (2): 115-135. doi: https://doi.org/10.1111/1758-2229.12810

13. Andreo-Martínez P, Rubio M, Veas A, Sánchez-Meca J, Martínez-González AE. A meta-analysis on the gut microbiota in children with autism. In press. J Autism Dev Disord 2021.

14. Lee I, Eriksson P, Fredriksson A, Buratovic S, Viberg H. Developmental neurotoxic effects of two pesticides: Behavior and neuroprotein studies on endosulfan and cypermethrin. Toxicology 2015, 335: 1-10. doi: https://doi.org/10.1016/j.tox.2015.06.01 
15. Roberts EM, English PB, Grether JK, Windham GC, Somberg L, Wolff C. Maternal Residence Near Agricultural Pesticide Applications and Autism Spectrum Disorders among Children in the California Central Valley. Environ Health Perspect 2007, 115 (10): 1482-1489. doi: https://doi.org/10.1289/ehp.10168

16. Gilden RC, Huffling K, Sattler B. Pesticides and Health Risks. J Obstet Gynecol Neonatal Nurs 2010, 39 (1): 103-110. doi: https://doi.org/10.1111/j.1552-6909.2009.01092.x

17. Sabarwal A, Kumar K, Singh RP. Hazardous effects of chemical pesticides on human health-Cancer and other associated disorders. Environ Toxicol Pharmacol 2018, 63: 103-114. doi: https://doi.org/10.1016/j.etap.2018.08.018

18. Regueiro J, López-Fernández $\mathrm{O}$, Rial-Otero R, Cancho-Grande B, Simal-Gándara J. A review on the fermentation of foods and the residues of pesticides-biotransformation of pesticides and effects on fermentation and food quality. Crit Rev Food Sci Nutr 2015, 55 (6): 839-863. doi: https://doi.org/10.1080/10408398.2012.677872

19. Sánchez-Bayo F, Goka K. Impacts of Pesticides on Honey Bees. Beekeeping and Bee Conservation Advances in Research. Chapter: 4: InTech Open Science; 2016. p. 77-97.

20. Frye CA, Bo E, Calamandrei G, Calzà L, Dessì-Fulgheri F, Fernández M, et al. Endocrine disrupters: a review of some sources, effects, and mechanisms of actions on behaviour and neuroendocrine systems. J Neuroendocrinol 2012, 24 (1): 144-159. doi: https://doi.org/10.1111/j.1365-2826.2011.02229.x

21. Jorsaraei SGA, Maliji G, Azadmehr A, Moghadamnia AA, Faraji AA. Immunotoxicity effects of carbaryl in vivo and in vitro. Environ Toxicol Pharmacol 2014, 38 (3): 838-844. doi: https://doi.org/10.1016/j.etap.2014.09.004

22. Page MJ, McKenzie JE, Bossuyt PM, Boutron I, Hoffmann TC, Mulrow CD, et al. The PRISMA 2020 statement: an updated guideline for reporting systematic reviews. BMJ 2021, 372: n71. doi: https://doi.org/10.1136/bmj.n71

23. Martínez-González AE, Andreo-Martínez P. The Role of Gut Microbiota in Gastrointestinal Symptoms of Children with ASD. Medicina 2019, 55 (8): $408 . \quad$ doi: https://doi.org/10.3390/medicina55080408

24. Sagiv SK, Harris MH, Gunier RB, Kogut KR, Harley KG, Deardorff J, et al. Prenatal Organophosphate Pesticide Exposure and Traits Related to Autism Spectrum Disorders in a Population Living in Proximity to Agriculture. Environ Health Perspect 2018, 126 (4): 047012. doi: https://doi.org/10.1289/EHP2580

25. Schmidt RJ, Kogan V, Shelton JF, Delwiche L, Hansen RL, Ozonoff S, et al. Combined Prenatal Pesticide Exposure and Folic Acid Intake in Relation to Autism Spectrum Disorder. Environ Health Perspect 2017, 125 (9): 097007. doi: https://doi.org/10.1289/EHP604

26. Shelton JF, Geraghty EM, Tancredi DJ, Delwiche LD, Schmidt RJ, Ritz B, et al. Neurodevelopmental disorders and prenatal residential proximity to agricultural pesticides: the CHARGE study. Environ Health Perspect 2014, 122 (10): 1103-1109. doi: https://doi.org/10.1289/ehp.1307044

27. Kimura-Kuroda J, Nagata I, Kuroda Y. Disrupting effects of hydroxy-polychlorinated biphenyl (PCB) congeners on neuronal development of cerebellar Purkinje cells: A possible causal factor for developmental brain disorders? Chemosphere 2007, 67 (9): S412-S420. doi: https://doi.org/10.1016/j.chemosphere.2006.05.137

28. Ferreira Gonzalez I, Urrutia G, Alonso-Coello P. Systematic reviews and meta-analysis: scientific rationale and interpretation. Rev Esp Cardiol 2011, 64 (8): 688-696. doi: https://doi.org/10.1016/j.recesp.2011.03.029

29. Lee S, McLaughlin R, Harnly M, Gunier R, Kreutzer R. Community exposures to airborne agricultural pesticides in California: ranking of inhalation risks. Environ Health Perspect 2002, 110 (12): 1175-1184. doi: https://doi.org/10.1289/ehp.021101175 
30. Loewenherz C, Fenske RA, Simcox NJ, Bellamy G, Kalman D. Biological monitoring of organophosphorus pesticide exposure among children of agricultural workers in central Washington State. Environ Health Perspect 1997, 105 (12): 1344-1353. doi: https://doi.org/10.1289/ehp.971051344

31. Cha YS, Kim H, Lee Y, Choi EH, Kim HI, Kim OH, et al. The relationship between serum ammonia level and neurologic complications in patients with acute glufosinate ammonium poisoning: A prospective observational study. Hum Exp Toxicol 2018, 37 (6): 571-579. doi: https://doi.org/10.1177/0960327117715902

32. Aris A, Leblanc S. Maternal and fetal exposure to pesticides associated to genetically modified foods in Eastern Townships of Quebec, Canada. Reprod Toxicol 2011, 31 (4): 528-533. doi: https://doi.org/10.1016/j.reprotox.2011.02.004

33. Feat-Vetel J, Larrigaldie V, Meyer-Dilhet G, Herzine A, Mougin C, Laugeray A, et al. Multiple effects of the herbicide glufosinate-ammonium and its main metabolite on neural stem cells from the subventricular zone of newborn mice. Neurotoxicology 2018, 69: 152-163. doi: https://doi.org/10.1016/j.neuro.2018.10.001

34. EPA. Endosulfan phase-out. http://www.epa.gov/pesticides/reregistration/endosulfan/endosulfanagreement.html. 2010.

35. Silva MH, Gammon D. An assessment of the developmental, reproductive, and neurotoxicity of endosulfan. Birth Defects Res B: Dev Reprod Toxicol 2009, 86 (1): 1-28. doi: https://doi.org/10.1002/bdrb.20183

36. ATSDR. Toxicological profile for endosulfan. 2013. http://www.atsdr.cdc.gov/toxprofiles/tp.asp?id=609\&tid=113

37. Cohen BI. GABA-transaminase, the liver and infantile autism. Med Hypotheses 2001, 57 (6): 673-674. doi: https://doi.org/10.1054/mehy.2001.1350

38. Shafer TJ, Meyer DA, Crofton KM. Developmental Neurotoxicity of Pyrethroid Insecticides: Critical Review and Future Research Needs. Environ Health Perspect 2005, 113 (2): 123-136. doi: https://doi.org/10.1289/ehp.7254

39. Rice DC. Behavioral Impairment Produced by Low-Level Postnatal PCB Exposure in Monkeys. Environ Res 1999, 80 (2): S113-S121. doi: https://doi.org/10.1006/enrs.1998.3917

40. Miyazaki W, Iwasaki T, Takeshita A, Kuroda Y, Koibuchi N. Polychlorinated biphenyls suppress thyroid hormone receptor-mediated transcription through a novel mechanism. J Biol Chem 2004, 279 (18): 18195-18202. doi: https://doi.org/10.1074/jbc.M310531200

41. Rietjens IMCM, den Besten C, Hanzlik RP, van Bladeren PJ. Cytochrome P450-Catalyzed Oxidation of Halobenzene Derivatives. Chem Res Toxicol 1997, 10 (6): 629-635. doi: https://doi.org/10.1021/tx9601061

42. Vidair CA. Age dependence of organophosphate and carbamate neurotoxicity in the postnatal rat: extrapolation to the human. Toxicol Appl Pharmacol 2004, 196 (2): 287-302. doi: https://doi.org/10.1016/j.taap.2003.12.016

43. Aluigi MG, Angelini C, Falugi C, Fossa R, Genever P, Gallus L, et al. Interaction between organophosphate compounds and cholinergic functions during development. Chem-Biol Interact 2005, 157-158: 305-316. doi: https://doi.org/10.1016/j.cbi.2005.10.037 